A. Zaigraev and A. Podraza-Karakulska (Toruń)

\title{
ON ASYMPTOTICS OF THE MAXIMUM LIKELIHOOD SCALE INVARIANT ESTIMATOR OF THE SHAPE PARAMETER OF THE GAMMA DISTRIBUTION
}

Abstract. The maximum likelihood scale invariant estimator of the shape parameter of the gamma distribution, proposed by the authors [Statist. Probab. Lett. 78 (2008)], is considered. The asymptotics of the mean square error of this estimator, with respect to that of the usual maximum likelihood estimator, is established.

1. Introduction. Let a sample $x=\left(x_{1}, \ldots, x_{n}\right)$ be drawn from the gamma distribution $\Gamma(\alpha, \sigma)$ with an unknown shape parameter $\alpha>0$ and an unknown scale parameter $\sigma>0$, whose density function has the form

$$
p(u ; \alpha, \sigma)=\frac{u^{\alpha-1} e^{-u / \sigma}}{\sigma^{\alpha} \Gamma(\alpha)}, \quad u>0 .
$$

Consider the problem of estimation of $\alpha$. One of the most popular estimators is the well-known maximum likelihood estimator (ML-estimator) (e.g. [4, Sections 9.3, 9.4], [6], [7], [8]). Let

$$
\mathbf{p}(x ; \alpha, \sigma)=\sigma^{-n \alpha}(\Gamma(\alpha))^{-n}\left(\prod_{j=1}^{n} x_{j}\right)^{\alpha-1} \exp \left(-\frac{1}{\sigma} \sum_{k=1}^{n} x_{k}\right)
$$

be the corresponding likelihood function. The ML-estimators $\alpha_{n}^{*}$ and $\sigma_{n}^{*}$ of $\alpha$ and $\sigma$, respectively, are determined by the equations

$$
\ln \sigma+\Psi(\alpha)=\left(\sum_{j=1}^{n} \ln x_{j}\right) / n, \quad \alpha-\sum_{k=1}^{n} x_{k} /(n \sigma)=0,
$$

2000 Mathematics Subject Classification: Primary 62F12; Secondary 62F10, 33B15.

Key words and phrases: gamma distribution, maximum likelihood estimator, maximum likelihood scale invariant estimator, mean square error, psi function. 
where $\Psi(\alpha)=\frac{d}{d \alpha} \ln \Gamma(\alpha)=(\ln \Gamma(\alpha))^{\prime}$ is the so-called Euler psi (digamma) function. Namely, $\alpha_{n}^{*}$ is the root of the equation

$$
g(\alpha)=T(x)
$$

with respect to $\alpha$, while

$$
\sigma_{n}^{*}=\frac{\bar{x}}{\alpha_{n}^{*}}
$$

where

$$
g(\alpha)=\ln \alpha-\Psi(\alpha), \quad T(x)=\ln \bar{x}-\frac{1}{n} \sum_{j=1}^{n} \ln x_{j}, \quad \bar{x}=\frac{1}{n} \sum_{k=1}^{n} x_{k} .
$$

Observe that the function $g$ is strictly decreasing and takes values in $(0, \infty)$ (e.g. Theorem 1 of [2]). Therefore, the estimator $\alpha_{n}^{*}$ is well-defined and unique.

Moreover,

$$
\mathrm{E} T(x)=g_{n}(\alpha)
$$

where

$$
g_{n}(\alpha)=\Psi(n \alpha)-\Psi(\alpha)-\ln n=g(\alpha)-g(n \alpha) .
$$

It is well-known (e.g. [10, Section 3.1], [11, Section 6.4]) that the limit distribution, as $n \rightarrow \infty$, of the random vector $n^{1 / 2}\left(\alpha_{n}^{*}-\alpha, \sigma_{n}^{*}-\sigma\right)$ is normal $\mathcal{N}\left(0, I^{-1}(\alpha, \sigma)\right)$, i.e. with zero mean vector and covariance matrix $I^{-1}(\alpha, \sigma)$, where $I(\alpha, \sigma)$ is the Fisher information matrix having the form

$$
I(\alpha, \sigma)=\left(\begin{array}{ll}
\Psi^{\prime}(\alpha) & 1 / \sigma \\
1 / \sigma & \alpha / \sigma^{2}
\end{array}\right) .
$$

This implies that the limit distribution of the random variable $n^{1 / 2}\left(\alpha_{n}^{*}-\alpha\right)$ is $\mathcal{N}\left(0, \kappa^{2}(\alpha)\right)$, where

$$
\kappa^{2}(\alpha)=\left(\psi^{\prime}(\alpha)-1 / \alpha\right)^{-1}=-1 / g^{\prime}(\alpha) .
$$

Note that the estimator $\alpha_{n}^{*}$ is scale invariant. The question arises: does there exist a better scale invariant estimator of $\alpha$ ? The positive answer is given in [13]. Estimating the shape parameter $\alpha$, one can consider $\sigma$ as a nuisance parameter. Therefore, it is natural to apply the maximum likelihood principle to the measure defined on the $\sigma$-algebra of scale invariant sets generated by the underlying gamma distribution. It is known (e.g. [9, Subsection 3.2.2], [12, Section 8.3]) that the density corresponding to this measure, with respect to that generated by $\mathcal{N}(0,1)$ distribution, is given as follows:

$$
\mathbf{q}(x ; \alpha)=\frac{\int_{0}^{\infty} t^{n-1} \mathbf{p}(t x ; \alpha, \sigma) d t}{\int_{0}^{\infty} t^{n-1} \mathbf{s}(t x) d t}=\frac{2 \pi^{n / 2} \Gamma(n \alpha)\left(\sum_{i=1}^{n} x_{i}^{2}\right)^{n / 2}\left(\prod_{i=1}^{n} x_{i}\right)^{\alpha-1}}{\Gamma(n / 2)(\Gamma(\alpha))^{n}\left(\sum_{i=1}^{n} x_{i}\right)^{n \alpha}},
$$


where

$$
\mathbf{s}(x)=(2 \pi)^{-n / 2} \exp \left(-\frac{1}{2} \sum_{k=1}^{n} x_{k}^{2}\right) .
$$

The maximum likelihood scale invariant estimator (IML-estimator) $\alpha_{n}^{* *}$ of $\alpha$ is defined as $\alpha_{n}^{* *} \in \arg \max _{\alpha>0} \mathbf{q}(x ; \alpha)$. By direct calculations one can find that $\alpha_{n}^{* *}$ is the root of the equation

$$
g_{n}(\alpha)=T(x)
$$

with respect to $\alpha$. Therefore, the IML-estimator $\alpha_{n}^{* *}$ coincides with that based on the method of moments. Of course, the estimator $\alpha_{n}^{* *}$ is scale invariant, well-defined and unique since the function $g_{n}$ is strictly decreasing and takes values in $(0, \infty)$ (see Lemma 1 of [13]).

It is worth noting that the scale invariance of the maximum likelihood estimator of a shape parameter is a quite common property in the case when the scale is also unknown. Indeed, consider the likelihood function

$$
\mathbf{p}(x ; \alpha, \sigma)=\sigma^{-n} \prod_{j=1}^{n} p\left(\sigma^{-1} x_{j} ; \alpha, 1\right),
$$

where $\alpha$ is a shape parameter taking values in $\left(\alpha_{-}, \alpha_{+}\right)$. Assume that

$$
\max _{\alpha \in\left(\alpha_{-}, \alpha_{+}\right), \sigma>0} \mathbf{p}(x ; \alpha, \sigma)=\max _{\alpha \in\left(\alpha_{-}, \alpha_{+}\right)} \max _{\sigma>0} \mathbf{p}(x ; \alpha, \sigma) .
$$

Let

$$
\widehat{\sigma}(x ; \alpha) \in \underset{\sigma>0}{\arg \max } \mathbf{p}(x ; \alpha, \sigma) .
$$

Observe that for any $\lambda>0$,

$$
\mathbf{p}(\lambda x ; \alpha, \sigma)=\lambda^{-n} \mathbf{p}(x ; \alpha, \sigma / \lambda),
$$

whence

$$
\widehat{\sigma}(\lambda x ; \alpha)=\lambda \widehat{\sigma}(x ; \alpha)
$$

Thus,

$$
\begin{aligned}
\left(\alpha_{n}^{*}, \sigma_{n}^{*}\right) & \in \underset{\alpha \in\left(\alpha_{-}, \alpha_{+}\right), \sigma>0}{\arg \max } \mathbf{p}(x ; \alpha, \sigma)=\underset{\alpha \in\left(\alpha_{-}, \alpha_{+}\right)}{\arg \max } \mathbf{p}(x ; \alpha, \widehat{\sigma}(x ; \alpha)) \\
& =\underset{\alpha \in\left(\alpha_{-}, \alpha_{+}\right)}{\arg \max }\left((\widehat{\sigma}(x ; \alpha))^{-n} \prod_{j=1}^{n} p\left((\widehat{\sigma}(x ; \alpha))^{-1} x_{j} ; \alpha, 1\right)\right) .
\end{aligned}
$$

It is evident that $\alpha_{n}^{*}(\lambda x)=\alpha_{n}^{*}(x)$, i.e. the estimator $\alpha_{n}^{*}$ is scale invariant. Therefore, it is reasonable to apply the method presented here also for other distributions.

In [13] it is shown that the IML-estimator is better than the ML-estimator in the sense that it has smaller bias and smaller variance. The main goal of 
this paper is to establish the asymptotics of the mean square error of $\alpha_{n}^{* *}$ compared to that of $\alpha_{n}^{*}$.

The paper is organized as follows. Section 2 deals with the asymptotic normality of the IML-estimator. The main result is established in Section 3 while all the auxiliary results are formulated and proved in the Appendix.

2. Asymptotic normality of the IML-estimator. As already noted, the limit distribution of $n^{1 / 2}\left(\alpha_{n}^{*}-\alpha\right)$, as $n \rightarrow \infty$, is $\mathcal{N}\left(0, \kappa^{2}(\alpha)\right)$, where $\kappa^{2}(\alpha)$ is defined by (2). Therefore, by Theorem 1.5 of [11, Section 5], the limit distribution of $n^{1 / 2}\left(g\left(\alpha_{n}^{*}\right)-g(\alpha)\right)$ is $\mathcal{N}\left(0, \kappa^{-2}(\alpha)\right)$. Since $g\left(\alpha_{n}^{*}\right)=g_{n}\left(\alpha_{n}^{* *}\right)$, the limit distribution of

$$
n^{1 / 2}\left(g_{n}\left(\alpha_{n}^{* *}\right)-g(\alpha)\right)=n^{1 / 2}\left(g\left(\alpha_{n}^{* *}\right)-g(\alpha)\right)-n^{1 / 2} g\left(n \alpha_{n}^{* *}\right)
$$

is also $\mathcal{N}\left(0, \kappa^{-2}(\alpha)\right)$.

Now observe that the well-known asymptotic formula

$$
\Psi(u)=\ln u-\frac{1}{2 u}-\sum_{k=1}^{\infty} \frac{B_{2 k}}{2 k u^{2 k}}, \quad u \rightarrow \infty
$$

(e.g. (6.3.18) of [1]), where $\left\{B_{k}\right\}$ are the so-called Bernoulli numbers, yields

$$
g(u)=\frac{1}{2 u}+\sum_{k=1}^{\infty} \frac{B_{2 k}}{2 k u^{2 k}}, \quad u \rightarrow \infty .
$$

From (4) it follows that $n^{1 / 2} g(n \alpha) \rightarrow 0$ as $n \rightarrow \infty$. Therefore, applying the Slutsky theorem we infer that the limit distribution of

$$
n^{1 / 2}\left(g\left(\alpha_{n}^{* *}\right)-g(\alpha)\right)-n^{1 / 2}\left(g\left(n \alpha_{n}^{* *}\right)-g(n \alpha)\right)
$$

is again $\mathcal{N}\left(0, \kappa^{-2}(\alpha)\right)$.

From (4) one can also obtain that for any given $\alpha$ and all sufficiently large $n$ we have $\left(n^{1 / 2} g(n \alpha)\right)^{\prime} \leq 1$. Let $\varepsilon>0$ be given. From Lemma 2 in the Appendix we get, as $n \rightarrow \infty$,

$$
\begin{aligned}
P\left(\left|n^{1 / 2} g\left(n \alpha_{n}^{* *}\right)-n^{1 / 2} g(n \alpha)\right| \geq \varepsilon\right) \leq P\left(\left|\alpha_{n}^{* *}-\alpha\right| \geq \varepsilon\right) \\
\quad=P\left(n^{1 / 2}\left|\alpha_{n}^{* *}-\alpha\right| \geq n^{1 / 2} \varepsilon\right) \leq c e^{-n \varepsilon^{2}\left(\Psi^{\prime}(\alpha)-1 / \alpha\right) / 8} \rightarrow 0 .
\end{aligned}
$$

Thus, as $n \rightarrow \infty$,

$$
n^{1 / 2}\left(g\left(n \alpha_{n}^{* *}\right)-g(n \alpha)\right) \rightarrow 0
$$

in probability. Again, the Slutsky theorem implies that the limit distribution of

$$
n^{1 / 2}\left(g\left(\alpha_{n}^{* *}\right)-g(\alpha)\right)
$$

is $\mathcal{N}\left(0, \kappa^{-2}(\alpha)\right)$. Finally, applying Theorem 1.5 of [11, Section 5$]$ leads to the following result: the limit distribution of $n^{1 / 2}\left(\alpha_{n}^{* *}-\alpha\right)$ is the same as that of $n^{1 / 2}\left(\alpha_{n}^{*}-\alpha\right)$, i.e. $\mathcal{N}\left(0, \kappa^{2}(\alpha)\right)$. 
3. Main result. Define

$$
R_{n}^{*}=\mathrm{E}\left(\alpha_{n}^{*}-\alpha\right)^{2}, \quad R_{n}^{* *}=\mathrm{E}\left(\alpha_{n}^{* *}-\alpha\right)^{2} .
$$

THEOREM. If a sample $x=\left(x_{1}, \ldots, x_{n}\right)$ is drawn from a $\Gamma(\alpha, \sigma)$ distribution, then

$$
n^{2}\left(R_{n}^{*}-R_{n}^{* *}\right)=D(\alpha)+o(1), \quad n \rightarrow \infty,
$$

where

$$
D(\alpha)=-\frac{3\left[g^{\prime}(\alpha) / \alpha+2 g^{\prime \prime}(\alpha)\right]}{4 \alpha\left(g^{\prime}(\alpha)\right)^{3}}>0 .
$$

Proof. Take a number $1 / 3<\delta<1 / 2$ and divide the sample space $(0, \infty)^{n}$ into $X_{n}=X_{n, \delta}$ and $X_{n}^{c}=(0, \infty)^{n} \backslash X_{n}$, where

$$
X_{n}=\left\{x:\left|\alpha_{n}^{*}-\alpha\right|<n^{-\delta},\left|\alpha_{n}^{* *}-\alpha\right|<n^{-\delta}\right\} .
$$

By the Cauchy-Schwarz inequality,

$$
n^{2} \mathrm{E}\left(\left(\alpha_{n}^{*}-\alpha\right)^{2} \mathbf{1}_{X_{n}^{c}}(x)\right) \leq\left(\mathrm{En}^{2}\left(\alpha_{n}^{*}-\alpha\right)^{4}\right)^{1 / 2}\left(n^{2} P\left(x \in X_{n}^{c}\right)\right)^{1 / 2} .
$$

In view of Lemmas 1 and 2 in the Appendix we obtain

$$
\begin{aligned}
n^{2} P\left(x \in X_{n}^{c}\right) \leq & n^{2} P\left(\left|\alpha_{n}^{*}-\alpha\right| \geq n^{-\delta}\right)+n^{2} P\left(\left|\alpha_{n}^{* *}-\alpha\right| \geq n^{-\delta}\right) \\
= & n^{2} P\left(n^{1 / 2}\left|\alpha_{n}^{*}-\alpha\right| \geq n^{1 / 2-\delta}\right) \\
& +n^{2} P\left(n^{1 / 2}\left|\alpha_{n}^{* *}-\alpha\right| \geq n^{1 / 2-\delta}\right) \rightarrow 0, \quad n \rightarrow \infty .
\end{aligned}
$$

Write for a moment $\xi_{n}=n^{1 / 2}\left(\alpha_{n}^{*}-\alpha\right)$. By Lemma 1 and integration by parts, for any $\gamma<\Psi^{\prime}(\alpha) / 8$ we have

$$
\begin{aligned}
\mathrm{E} e^{\gamma \xi_{n}^{2}} & =-\int_{0}^{\infty} e^{\gamma u^{2}} d P\left(\left|\xi_{n}\right| \geq u\right)=1+2 \gamma \int_{0}^{\infty} u e^{\gamma u^{2}} P\left(\left|\xi_{n}\right| \geq u\right) d u \\
& \leq 1+4 \gamma \int_{0}^{\infty} u e^{\left(\gamma-\Psi^{\prime}(\alpha) / 8\right) u^{2}} d u<\infty
\end{aligned}
$$

Since for any $\gamma>0$,

$$
\gamma^{2} z^{4}<e^{\gamma z^{2}}, \quad z>0
$$

we deduce that for any $0<\gamma<\Psi^{\prime}(\alpha) / 8$,

$$
\mathrm{E}\left[n^{1 / 2}\left(\alpha_{n}^{*}-\alpha\right)\right]^{4}=\mathrm{E}\left|\xi_{n}\right|^{4}<\gamma^{-2} \mathrm{E} e^{\gamma \xi_{n}^{2}}<\infty .
$$

Therefore,

$$
n^{2} \mathrm{E}\left(\left(\alpha_{n}^{*}-\alpha\right)^{2} \mathbf{1}_{X_{n}^{c}}(x)\right) \rightarrow 0, \quad n \rightarrow \infty .
$$

A similar reasoning with an application of Lemma 2 leads to

$$
n^{2} \mathrm{E}\left(\left(\alpha_{n}^{* *}-\alpha\right)^{2} \mathbf{1}_{X_{n}^{c}}(x)\right) \rightarrow 0, \quad n \rightarrow \infty .
$$

Thus, in order to prove the theorem it is enough to consider only the case $x \in X_{n}$ as $n \rightarrow \infty$, which we assume until the end of the proof. 
Applying the Taylor formula and formula (4) yields

$$
\begin{aligned}
g\left(\alpha_{n}^{* *}\right)-g\left(\alpha_{n}^{*}\right) & =g\left(\alpha_{n}^{* *}\right)-g_{n}\left(\alpha_{n}^{* *}\right)=g\left(n \alpha_{n}^{* *}\right) \\
& =g(n \alpha)+g^{\prime}(n \alpha) n\left(\alpha_{n}^{* *}-\alpha\right)+O\left(n^{-1-2 \delta}\right) \\
& =\frac{1}{2 n \alpha}-\frac{\alpha_{n}^{* *}-\alpha}{2 n \alpha^{2}}+O\left(n^{-1-2 \delta}\right) .
\end{aligned}
$$

On the other hand, using the Taylor formula one can obtain

(8) $g\left(\alpha_{n}^{* *}\right)-g\left(\alpha_{n}^{*}\right)=g^{\prime}\left(\alpha_{n}^{*}\right)\left(\alpha_{n}^{* *}-\alpha_{n}^{*}\right)+\frac{g^{\prime \prime}\left(\alpha_{n}^{*}\right)}{2}\left(\alpha_{n}^{* *}-\alpha_{n}^{*}\right)^{2}+O\left(\left|\alpha_{n}^{* *}-\alpha_{n}^{*}\right|^{3}\right)$.

Similarly,

$$
\begin{aligned}
g^{\prime}\left(\alpha_{n}^{*}\right) & =g^{\prime}(\alpha)+g^{\prime \prime}(\alpha)\left(\alpha_{n}^{*}-\alpha\right)+O\left(n^{-2 \delta}\right), \\
g^{\prime \prime}\left(\alpha_{n}^{*}\right) & =g^{\prime \prime}(\alpha)+O\left(n^{-\delta}\right) .
\end{aligned}
$$

By substituting (9) and (10) into (8), we have

$$
\begin{aligned}
& g\left(\alpha_{n}^{* *}\right)-g\left(\alpha_{n}^{*}\right) \\
= & g^{\prime}(\alpha)\left(\alpha_{n}^{* *}-\alpha_{n}^{*}\right)\left(1+\frac{g^{\prime \prime}(\alpha)}{g^{\prime}(\alpha)}\left(\alpha_{n}^{*}-\alpha\right)+\frac{g^{\prime \prime}(\alpha)}{2 g^{\prime}(\alpha)}\left(\alpha_{n}^{* *}-\alpha_{n}^{*}\right)+O\left(n^{-2 \delta}\right)\right) .
\end{aligned}
$$

Comparing (7) and (11), we get

$$
g^{\prime}(\alpha)\left(\alpha_{n}^{* *}-\alpha_{n}^{*}\right)\left(1+L_{n}(\alpha)+O\left(n^{-2 \delta}\right)\right)=\frac{1}{2 n \alpha}-\frac{\alpha_{n}^{* *}-\alpha}{2 n \alpha^{2}}+O\left(n^{-1-2 \delta}\right),
$$

where

$$
L_{n}(\alpha)=\frac{g^{\prime \prime}(\alpha)}{2 g^{\prime}(\alpha)}\left[2\left(\alpha_{n}^{*}-\alpha\right)+\alpha_{n}^{* *}-\alpha_{n}^{*}\right]=\frac{g^{\prime \prime}(\alpha)}{2 g^{\prime}(\alpha)}\left[\alpha_{n}^{*}-\alpha+\alpha_{n}^{* *}-\alpha\right] .
$$

From (12) it follows that

$$
\begin{aligned}
\alpha_{n}^{* *} & -\alpha_{n}^{*}=\frac{\alpha-\left(\alpha_{n}^{* *}-\alpha\right)}{2 n \alpha^{2} g^{\prime}(\alpha)\left(1+L_{n}(\alpha)\right)}+O\left(n^{-1-2 \delta}\right) \\
& =\frac{\alpha-\left(\alpha_{n}^{* *}-\alpha\right)}{2 n \alpha^{2} g^{\prime}(\alpha)}\left(1-\frac{g^{\prime \prime}(\alpha)}{2 g^{\prime}(\alpha)}\left[\alpha_{n}^{*}-\alpha+\alpha_{n}^{* *}-\alpha\right]\right)+O\left(n^{-1-2 \delta}\right) .
\end{aligned}
$$

This can be rewritten as

$$
\begin{aligned}
\alpha_{n}^{* *}-\alpha= & \alpha_{n}^{*}-\alpha+\frac{1}{2 n \alpha g^{\prime}(\alpha)}-\frac{\alpha_{n}^{* *}-\alpha}{2 n \alpha^{2} g^{\prime}(\alpha)}-\frac{g^{\prime \prime}(\alpha)\left(\alpha_{n}^{* *}-\alpha\right)}{4 n \alpha\left(g^{\prime}(\alpha)\right)^{2}} \\
& -\frac{g^{\prime \prime}(\alpha)\left(\alpha_{n}^{*}-\alpha\right)}{4 n \alpha\left(g^{\prime}(\alpha)\right)^{2}}+O\left(n^{-1-2 \delta}\right) .
\end{aligned}
$$

Therefore, we obtain

$$
A_{n}(\alpha)\left(\alpha_{n}^{* *}-\alpha\right)=B_{n}(\alpha)\left(\alpha_{n}^{*}-\alpha\right)+C_{n}(\alpha)+O\left(n^{-1-2 \delta}\right),
$$


where

$$
\begin{aligned}
& A_{n}(\alpha)=1+\frac{1}{2 n \alpha^{2} g^{\prime}(\alpha)}+\frac{g^{\prime \prime}(\alpha)}{4 n \alpha\left(g^{\prime}(\alpha)\right)^{2}} \\
& B_{n}(\alpha)=1-\frac{g^{\prime \prime}(\alpha)}{4 n \alpha\left(g^{\prime}(\alpha)\right)^{2}}, \quad C_{n}(\alpha)=\frac{1}{2 n \alpha g^{\prime}(\alpha)}
\end{aligned}
$$

Set $b_{n}(\alpha)=\mathrm{E}\left(\alpha_{n}^{*}-\alpha\right)$. Applying the Taylor formula, we obtain

$$
g\left(\alpha_{n}^{*}\right)-g(\alpha)=g^{\prime}(\alpha)\left(\alpha_{n}^{*}-\alpha\right)+\frac{g^{\prime \prime}(\alpha)}{2}\left(\alpha_{n}^{*}-\alpha\right)^{2}+O\left(n^{-3 \delta}\right) .
$$

Further,

$$
\mathrm{E}\left(g\left(\alpha_{n}^{*}\right)-g(\alpha)\right)=g^{\prime}(\alpha) b_{n}(\alpha)+\frac{g^{\prime \prime}(\alpha)}{2} R_{n}^{*}+O\left(n^{-3 \delta}\right)
$$

On the other hand, by (3) and (1) we have

$$
\mathrm{E}\left(g\left(\alpha_{n}^{*}\right)-g(\alpha)\right)=-g(n \alpha) .
$$

Comparing (15) and (16) and making use of (4) we get

$$
b_{n}(\alpha)=-\frac{1}{2 n \alpha g^{\prime}(\alpha)}-\frac{g^{\prime \prime}(\alpha)}{2 g^{\prime}(\alpha)} R_{n}^{*}+O\left(n^{-3 \delta}\right) .
$$

Observe that from (14) it follows that

$$
A_{n}^{2}(\alpha) R_{n}^{* *}=B_{n}^{2}(\alpha) R_{n}^{*}+C_{n}^{2}(\alpha)+2 B_{n}(\alpha) C_{n}(\alpha) b_{n}(\alpha)+O\left(n^{-2-2 \delta}\right),
$$

or, by substituting (17),

$$
\begin{aligned}
A_{n}^{2}(\alpha) R_{n}^{* *}= & B_{n}^{2}(\alpha) R_{n}^{*}+C_{n}^{2}(\alpha)-2 B_{n}(\alpha) C_{n}^{2}(\alpha) \\
& -B_{n}(\alpha) C_{n}(\alpha) \frac{g^{\prime \prime}(\alpha)}{g^{\prime}(\alpha)} R_{n}^{*}+O\left(n^{-1-3 \delta}\right) .
\end{aligned}
$$

Therefore,

$$
\begin{aligned}
n^{2}\left(R_{n}^{* *}-R_{n}^{*}\right)= & n^{2} R_{n}^{*}\left(\frac{B_{n}^{2}(\alpha)-B_{n}(\alpha) C_{n}(\alpha) g^{\prime \prime}(\alpha) / g^{\prime}(\alpha)}{A_{n}^{2}(\alpha)}-1\right) \\
& +n^{2} \frac{C_{n}^{2}(\alpha)-2 B_{n}(\alpha) C_{n}^{2}(\alpha)}{A_{n}^{2}(\alpha)}+o(1) .
\end{aligned}
$$

Since the limit distribution of $n^{1 / 2}\left(\alpha_{n}^{*}-\alpha\right)$, as $n \rightarrow \infty$, is $\mathcal{N}\left(0, \kappa^{2}(\alpha)\right)$, and

$$
\mathrm{E}\left[n^{1 / 2}\left(\alpha_{n}^{*}-\alpha\right)\right]^{4}<\infty
$$

(see (6)), by the moment continuity theorem (e.g. Theorem 4 of $[5$, Section 1 , $\S 6])$ we obtain

$$
\mathrm{E}\left[n^{1 / 2}\left(\alpha_{n}^{*}-\alpha\right)\right]^{2} \rightarrow \kappa^{2}(\alpha)=-1 / g^{\prime}(\alpha), \quad n \rightarrow \infty .
$$

Thus,

$$
n R_{n}^{*}=-1 / g^{\prime}(\alpha)+o(1), \quad n \rightarrow \infty
$$


The proof is finished by substituting (19) into (18) and taking into account Lemma 3 below, formula (4) and the relations

$$
\begin{aligned}
n\left(\frac{B_{n}^{2}(\alpha)-B_{n}(\alpha) C_{n}(\alpha) g^{\prime \prime}(\alpha) / g^{\prime}(\alpha)}{A_{n}^{2}(\alpha)}-1\right) & =-\frac{3 g^{\prime \prime}(\alpha)}{2 \alpha\left(g^{\prime}(\alpha)\right)^{2}}-\frac{1}{\alpha^{2} g^{\prime}(\alpha)}+o(1), \\
n^{2} \frac{C_{n}^{2}(\alpha)-2 B_{n}(\alpha) C_{n}^{2}(\alpha)}{A_{n}^{2}(\alpha)} & =-\frac{1}{4 \alpha^{2}\left(g^{\prime}(\alpha)\right)^{2}}+o(1) .
\end{aligned}
$$

\section{Appendix}

LEMMA 1. If a sample $x=\left(x_{1}, \ldots, x_{n}\right)$ is drawn from a $\Gamma(\alpha, \sigma)$ distribution, then for any $z>0$ and any $c>1$ there exists $N=N(z, c)$ such that for all $n \geq N$,

$$
P\left(n^{1 / 2}\left|\alpha_{n}^{*}-\alpha\right| \geq z\right) \leq c e^{-\Psi^{\prime}(\alpha) z^{2} / 8} .
$$

Proof. Consider the random function (cf. [5, §23])

$$
Z_{n}(\beta)=\frac{\mathbf{p}(x ; \alpha+\beta, 1)}{\mathbf{p}(x ; \alpha, 1)}, \quad \beta>-\alpha .
$$

Fix $z>0$. Since

$$
\left\{\left|\alpha_{n}^{*}-\alpha\right| \geq z\right\}=\left\{\sup _{|\beta| \geq z} Z_{n}(\beta) \geq \sup _{|\beta| \leq z} Z_{n}(\beta)\right\} \subset\left\{\sup _{|\beta| \geq z} Z_{n}(\beta) \geq Z_{n}(0)=1\right\}
$$

we have

$$
P\left(n^{1 / 2}\left|\alpha_{n}^{*}-\alpha\right| \geq z\right) \leq P\left(\sup _{\beta \in B} Z_{n}(\beta) \geq 1\right),
$$

where

$$
B=(-\alpha,-z / \sqrt{n}] \cup[z / \sqrt{n}, \infty) .
$$

From the Markov inequality, we obtain

$$
P\left(\sup _{\beta \in B} Z_{n}(\beta) \geq 1\right) \leq \mathrm{E}\left(\sup _{\beta \in B} Z_{n}(\beta)\right)^{1 / 2}=\mathrm{E} \sup _{\beta \in B} Z_{n}^{1 / 2}(\beta) .
$$

Then

$$
\mathrm{E} \sup _{\beta \in B} Z_{n}^{1 / 2}(\beta) \leq\left(\sup _{\beta \in B} \int_{0}^{\infty} p^{1 / 2}(u ; \alpha, 1) p^{1 / 2}(u ; \alpha+\beta, 1) d u\right)^{n} .
$$

Simple calculation yields

$$
\int_{0}^{\infty} p^{1 / 2}(u ; \alpha, 1) p^{1 / 2}(u ; \alpha+\beta, 1) d u=\frac{\Gamma(\alpha+\beta / 2)}{(\Gamma(\alpha) \Gamma(\alpha+\beta))^{1 / 2}} .
$$

Let us investigate the properties of the function

$$
\varrho(u)=\frac{\Gamma(\alpha+u / 2)}{(\Gamma(\alpha) \Gamma(\alpha+u))^{1 / 2}}, \quad u>-\alpha .
$$


Clearly,

$$
(\ln \varrho(u))^{\prime}=\frac{1}{2}\left[\Psi\left(\alpha+\frac{u}{2}\right)-\Psi(\alpha+u)\right] .
$$

Since $\Psi(u)$ is increasing, it follows that $\varrho(u)$ is increasing in $(-\alpha, 0)$ and decreasing in $(0, \infty)$ with maximum equal to 1 at $u=0$. Furthermore,

$$
\ln \varrho(-u)<\ln \varrho(u), \quad 0<u<\alpha .
$$

Indeed, the function $\widetilde{\varrho}(u)=\ln \varrho(u)-\ln \varrho(-u)$ has $\widetilde{\varrho}(0)=0$ and

$$
\widetilde{\varrho}(u)=\frac{1}{2}\left[\Psi\left(\alpha+\frac{u}{2}\right)+\Psi\left(\alpha-\frac{u}{2}\right)-\Psi(\alpha+u)-\Psi(\alpha-u)\right]>0
$$

since the function $\Psi(\alpha+z)+\Psi(\alpha-z)$ is decreasing because $(\Psi(\alpha+z)$ $+\Psi(\alpha-z))^{\prime}<0$ in view of $\Psi^{\prime}(\alpha+z)<\Psi^{\prime}(\alpha-z)$ for $z>0\left(\Psi^{\prime}(u)\right.$ is decreasing).

Hence,

$$
\sup _{\beta \in B} \varrho(\beta)=\varrho\left(\frac{z}{\sqrt{n}}\right) .
$$

By the Taylor formula, for all sufficiently large $n$ we obtain

$$
\varrho\left(\frac{z}{\sqrt{n}}\right)=1+\frac{\varrho^{\prime \prime}(0)}{2} \cdot \frac{z^{2}}{n}+o\left(n^{-1}\right) .
$$

But from (20) it follows that

$$
\varrho^{\prime}(u)=\frac{\varrho(u)}{2}\left[\Psi\left(\alpha+\frac{u}{2}\right)-\Psi(\alpha+u)\right] .
$$

Therefore,

$$
\varrho^{\prime \prime}(u)=\frac{\varrho^{\prime}(u)}{2}\left[\Psi\left(\alpha+\frac{u}{2}\right)-\Psi(\alpha+u)\right]+\frac{\varrho(u)}{2}\left[\frac{1}{2} \Psi^{\prime}\left(\alpha+\frac{u}{2}\right)-\Psi^{\prime}(\alpha+u)\right]
$$

and

$$
\varrho^{\prime \prime}(0)=-\frac{1}{4} \Psi^{\prime}(\alpha)<0 .
$$

Substitution into (21) yields

$$
\varrho\left(\frac{z}{\sqrt{n}}\right)=1-\frac{\Psi^{\prime}(\alpha) z^{2}}{8 n}+o\left(n^{-1}\right) .
$$

Thus, for any $c>1$ and all sufficiently large $n$ we obtain

$$
\begin{aligned}
\left(\sup _{\beta \in B} \int_{0}^{\infty} p^{1 / 2}(u ; \alpha, 1) p^{1 / 2}(u ; \alpha+\beta, 1) d u\right)^{n} & \\
= & \left(1-\frac{\Psi^{\prime}(\alpha) z^{2}}{8 n}+o\left(n^{-1}\right)\right)^{n} \leq c e^{-\Psi^{\prime}(\alpha) z^{2} / 8} .
\end{aligned}
$$

The proof is complete. 
Lemma 2. If a sample $x=\left(x_{1}, \ldots, x_{n}\right)$ is drawn from a $\Gamma(\alpha, \sigma)$ distribution, then for any $z>0$ and any $c>1$ there exists $N=N(z, c)$ such that for all $n \geq N$,

$$
P\left(n^{1 / 2}\left|\alpha_{n}^{* *}-\alpha\right| \geq z\right) \leq c e^{-\left(\Psi^{\prime}(\alpha)-1 / \alpha\right) z^{2} / 8} .
$$

Proof. The method of establishing this result is similar to that of proving Lemma 1. Consider the random function

$$
\begin{aligned}
Z_{n}(\beta) & =\frac{\mathbf{q}(x ; \alpha+\beta)}{\mathbf{q}(x ; \alpha)}=\frac{\Gamma(n(\alpha+\beta))\left(\prod_{i=1}^{n} x_{i}\right)^{\alpha+\beta-1}}{(\Gamma(\alpha+\beta))^{n}\left(\sum_{i=1}^{n} x_{i}\right)^{n(\alpha+\beta)}} \cdot \frac{(\Gamma(\alpha))^{n}\left(\sum_{i=1}^{n} x_{i}\right)^{n \alpha}}{\Gamma(n \alpha)\left(\prod_{i=1}^{n} x_{i}\right)^{\alpha-1}} \\
& =\frac{\Gamma(n(\alpha+\beta))(\Gamma(\alpha))^{n}}{(\Gamma(\alpha+\beta))^{n} \Gamma(n \alpha)} \cdot \frac{\left(\prod_{i=1}^{n} x_{i}\right)^{\beta}}{\left(\sum_{i=1}^{n} x_{i}\right)^{n \beta}} .
\end{aligned}
$$

Then

$$
Z_{n}^{1 / 2}(\beta)=\frac{(\Gamma(n(\alpha+\beta)))^{1 / 2}(\Gamma(\alpha))^{n / 2}}{(\Gamma(\alpha+\beta))^{n / 2}(\Gamma(n \alpha))^{1 / 2}} \cdot \frac{\left(\prod_{i=1}^{n} x_{i}\right)^{\beta / 2}}{\left(\sum_{i=1}^{n} x_{i}\right)^{n \beta / 2}} .
$$

Therefore,

$$
\begin{aligned}
\mathrm{E} Z_{n}^{1 / 2}(\beta)= & \frac{(\Gamma(n(\alpha+\beta)))^{1 / 2}}{(\Gamma(\alpha+\beta))^{n / 2}(\Gamma(n \alpha))^{1 / 2}(\Gamma(\alpha))^{n / 2}} \\
& \cdot \int_{0}^{\infty} \ldots \int_{0}^{\infty} \frac{\left(u_{1} \ldots u_{n}\right)^{\alpha+\beta / 2-1}}{\left(u_{1}+\cdots+u_{n}\right)^{n \beta / 2}} e^{-\left(u_{1}+\cdots+u_{n}\right)} d u_{1} \ldots d u_{n} .
\end{aligned}
$$

The change of variables $v_{1}=u_{1}, \ldots, v_{n-1}=u_{n-1}, v_{n}=u_{1}+\cdots+u_{n}$ yields

$$
\begin{aligned}
\mathrm{E} Z_{n}^{1 / 2}(\beta)= & \frac{(\Gamma(n(\alpha+\beta)))^{1 / 2}}{(\Gamma(\alpha+\beta))^{n / 2}(\Gamma(n \alpha))^{1 / 2}(\Gamma(\alpha))^{n / 2}} \\
& \cdot \int_{0}^{\infty} \ldots \int_{0}^{\infty} \int_{v_{1}+\cdots+v_{n-1}}^{\infty} \frac{\left[v_{1} \ldots v_{n-1}\left(v_{n}-v_{1}-\cdots-v_{n-1}\right)\right]^{\alpha+\beta / 2-1}}{v_{n}^{n \beta / 2}} \\
& \cdot e^{-v_{n}} d v_{n} \ldots d v_{2} d v_{1} .
\end{aligned}
$$

The next change of variables $v_{1}=v_{n} z_{1}, \ldots, v_{n-1}=v_{n} z_{n-1}$ gives

$$
\begin{aligned}
\mathrm{E} Z_{n}^{1 / 2}(\beta)= & \frac{(\Gamma(n(\alpha+\beta)))^{1 / 2}}{(\Gamma(\alpha+\beta))^{n / 2}(\Gamma(n \alpha))^{1 / 2}(\Gamma(\alpha))^{n / 2}} \int_{0}^{\infty} v_{n}^{n \alpha-1} e^{-v_{n}} d v_{n} \\
& \cdot \int_{A} \ldots \int\left[z_{1} \ldots z_{n-1}\left(1-z_{1}-\cdots-z_{n-1}\right)\right]^{\alpha+\beta / 2-1} d z_{1} \ldots d z_{n-1} \\
= & \frac{(\Gamma(n(\alpha+\beta)))^{1 / 2}(\Gamma(n \alpha))^{1 / 2}}{(\Gamma(\alpha+\beta))^{n / 2}(\Gamma(\alpha))^{n / 2}} \\
& \cdot \int_{A} \ldots \int\left[z_{1} \ldots z_{n-1}\left(1-z_{1}-\cdots-z_{n-1}\right)\right]^{\alpha+\beta / 2-1} d z_{1} \ldots d z_{n-1}
\end{aligned}
$$




$$
\begin{aligned}
= & \frac{(\Gamma(n(\alpha+\beta)))^{1 / 2}(\Gamma(n \alpha))^{1 / 2}}{(\Gamma(\alpha+\beta))^{n / 2}(\Gamma(\alpha))^{n / 2}} \int_{0}^{1} z_{1}^{\alpha+\beta / 2-1} d z_{1} \int_{0}^{1-z_{1}} z_{2}^{\alpha+\beta / 2-1} d z_{2} \ldots \\
& \cdots \quad \int_{0}^{1-z_{1}-\cdots-z_{n-2}} z_{n-1}^{\alpha+\beta / 2-1}\left(1-z_{1}-\cdots-z_{n-2}-z_{n-1}\right)^{\alpha+\beta / 2-1} d z_{n-1} \\
& \quad \frac{(\Gamma(n(\alpha+\beta)))^{1 / 2}(\Gamma(n \alpha))^{1 / 2}}{(\Gamma(\alpha+\beta))^{n / 2}(\Gamma(\alpha))^{n / 2}} I_{n},
\end{aligned}
$$

where

$$
A=\left\{\left(z_{1}, \ldots, z_{n-1}\right): z_{1}>0, \ldots, z_{n-1}>0, z_{1}+\cdots+z_{n-1}<1\right\} .
$$

Now we calculate $I_{n}$. Observe that

$$
\int_{0}^{a} u^{y}(a-u)^{z} d u=a^{y+z+1} B(y+1, z+1), \quad y, z>-1,
$$

where $B(\cdot, \cdot)$ is the beta-function. Then

$$
\begin{aligned}
\int_{0}^{1-z_{1}-\cdots-z_{n-2}} z_{n-1}^{\alpha+\beta / 2-1} & \left(1-z_{1}-\cdots-z_{n-2}-z_{n-1}\right)^{\alpha+\beta / 2-1} d z_{n-1} \\
= & \left(1-z_{1}-\cdots-z_{n-2}\right)^{2 \alpha+\beta-1} B(\alpha+\beta / 2, \alpha+\beta / 2) .
\end{aligned}
$$

Therefore,

$$
\begin{aligned}
I_{n}= & B(\alpha+\beta / 2, \alpha+\beta / 2) \int_{0}^{1} z_{1}^{\alpha+\beta / 2-1} d z_{1} \int_{0}^{1-z_{1}} z_{2}^{\alpha+\beta / 2-1} d z_{2} \ldots \\
& \ldots \int_{0}^{1-z_{1}-\cdots-z_{n-3}} z_{n-2}^{\alpha+\beta / 2-1}\left(1-z_{1}-\cdots-z_{n-3}-z_{n-2}\right)^{2 \alpha+\beta-1} d z_{n-2} .
\end{aligned}
$$

Again,

$$
\begin{gathered}
\int_{0}^{1-z_{1}-\cdots-z_{n-3}} z_{n-2}^{\alpha+\beta / 2-1}\left(1-z_{1}-\cdots-z_{n-3}-z_{n-2}\right)^{2 \alpha+\beta-1} d z_{n-2} \\
=\left(1-z_{1}-\cdots-z_{n-3}\right)^{3 \alpha+3 \beta / 2-1} B(\alpha+\beta / 2,2(\alpha+\beta / 2)),
\end{gathered}
$$

and we obtain

$$
\begin{aligned}
I_{n}= & B(\alpha+\beta / 2, \alpha+\beta / 2) B(\alpha+\beta / 2,2(\alpha+\beta / 2)) \\
& \cdot \int_{0}^{1} z_{1}^{\alpha+\beta / 2-1} d z_{1} \int_{0}^{1-z_{1}} z_{2}^{\alpha+\beta / 2-1} d z_{2} \ldots \\
& \ldots \quad \int_{0}^{1-z_{1}-\cdots-z_{n-4}} z_{n-3}^{\alpha+\beta / 2-1}\left(1-z_{1}-\cdots-z_{n-4}-z_{n-3}\right)^{3 \alpha+3 \beta / 2-1} d z_{n-3} .
\end{aligned}
$$


Repeating this calculation, we get

$$
\begin{aligned}
I_{n}= & B(\alpha+\beta / 2, \alpha+\beta / 2) B(\alpha+\beta / 2,2(\alpha+\beta / 2)) \\
& \ldots B(\alpha+\beta / 2,(n-1)(\alpha+\beta / 2)) \\
= & \frac{(\Gamma(\alpha+\beta / 2))^{n}}{\Gamma(n(\alpha+\beta / 2))} .
\end{aligned}
$$

Thus,

$$
\mathrm{E} Z_{n}^{1 / 2}(\beta)=\frac{(\Gamma(n(\alpha+\beta)))^{1 / 2}(\Gamma(\alpha+\beta / 2))^{n}(\Gamma(n \alpha))^{1 / 2}}{(\Gamma(\alpha+\beta))^{n / 2} \Gamma(n(\alpha+\beta / 2))(\Gamma(\alpha))^{n / 2}}=\left(\Delta_{n}(\beta)\right)^{n} .
$$

Let us investigate the properties of the function

$$
\Delta_{n}(u)=\frac{\Gamma(\alpha+u / 2)(\Gamma(n(\alpha+u)))^{1 /(2 n)}(\Gamma(n \alpha))^{1 /(2 n)}}{(\Gamma(\alpha+u))^{1 / 2}(\Gamma(\alpha))^{1 / 2}(\Gamma(n(\alpha+u / 2)))^{1 / n}}, \quad u>-\alpha .
$$

Clearly,

$$
\begin{aligned}
\ln \Delta_{n}(u)= & \ln \Gamma(\alpha+u / 2)-\frac{1}{2} \ln \Gamma(\alpha+u)-\frac{1}{2} \ln \Gamma(\alpha) \\
& +\frac{1}{2 n} \ln \Gamma(n(\alpha+u))+\frac{1}{2 n} \ln \Gamma(n \alpha) \\
& -\frac{1}{n} \ln \Gamma(n(\alpha+u / 2)), \\
\left(\ln \Delta_{n}(u)\right)^{\prime}= & \frac{1}{2} \Psi(\alpha+u / 2)-\frac{1}{2} \Psi(\alpha+u)+\frac{1}{2} \Psi(n(\alpha+u)) \\
& -\frac{1}{2} \Psi(n(\alpha+u / 2)) \\
= & \frac{1}{2}\left[g_{n}(\alpha+u)-g_{n}(\alpha+u / 2)\right] .
\end{aligned}
$$

Since $g_{n}(u)$ is decreasing (see Lemma 1 of [13]), it follows that $\Delta_{n}(u)$ is increasing in $(-\alpha, 0)$ and decreasing in $(0, \infty)$ with maximum equal to 1 at $u=0$. Furthermore,

$$
\ln \Delta_{n}(-u)<\ln \Delta_{n}(u), \quad 0<u<\alpha .
$$

Indeed, the function $\widetilde{\Delta}_{n}(u)=\ln \Delta_{n}(u)-\ln \Delta_{n}(-u)$ has $\widetilde{\Delta}_{n}(0)=0$ and

$$
\widetilde{\Delta}_{n}^{\prime}(u)=\frac{1}{2}\left[g_{n}(\alpha+u)+g_{n}(\alpha-u)-g_{n}\left(\alpha+\frac{u}{2}\right)-g_{n}\left(\alpha-\frac{u}{2}\right)\right]>0
$$

since the function $\zeta(z)=g_{n}(\alpha+z)+g_{n}(\alpha-z)$ is increasing because $\left(g_{n}(\alpha+z)+g_{n}(\alpha-z)\right)^{\prime}>0$ in view of $g_{n}^{\prime}(\alpha+z)>g_{n}^{\prime}(\alpha-z)$ for $z>0\left(g_{n}^{\prime}(u)\right.$ is increasing).

Hence,

$$
\sup _{\beta \in B} \Delta_{n}(\beta)=\Delta_{n}\left(\frac{z}{\sqrt{n}}\right) .
$$


Now observe that from (22) it follows that

$$
\begin{aligned}
\Delta_{n}^{\prime}(u)= & \frac{\Delta_{n}(u)}{2}\left[g_{n}(\alpha+u)-g_{n}\left(\alpha+\frac{u}{2}\right)\right], \\
\Delta_{n}^{\prime \prime}(u)= & \frac{\Delta_{n}^{\prime}(u)}{2}\left[g_{n}(\alpha+u)-g_{n}\left(\alpha+\frac{u}{2}\right)\right] \\
& +\frac{\Delta_{n}(u)}{2}\left[g_{n}^{\prime}(\alpha+u)-\frac{1}{2} g_{n}^{\prime}\left(\alpha+\frac{u}{2}\right)\right], \\
\Delta_{n}^{\prime \prime \prime}(u)= & \frac{\Delta_{n}^{\prime \prime}(u)}{2}\left[g_{n}(\alpha+u)-g_{n}\left(\alpha+\frac{u}{2}\right)\right] \\
& +\Delta_{n}^{\prime}(u)\left[g_{n}^{\prime}(\alpha+u)-\frac{1}{2} g_{n}^{\prime}\left(\alpha+\frac{u}{2}\right)\right] \\
& +\frac{\Delta_{n}(u)}{2}\left[g_{n}^{\prime \prime}(\alpha+u)-\frac{1}{4} g_{n}^{\prime \prime}\left(\alpha+\frac{u}{2}\right)\right] .
\end{aligned}
$$

Therefore,

$$
\Delta_{n}^{(k)}(0)=\frac{1}{2}\left(1-\frac{1}{2^{k-1}}\right) g_{n}^{(k-1)}(\alpha), \quad k \geq 1 .
$$

Since for all sufficiently large $n$ (see (4)),

$$
g_{n}^{(k-1)}(\alpha)=g^{(k-1)}(\alpha)+O\left(n^{-1}\right),
$$

by the Taylor formula we obtain, for all sufficiently large $n$,

$$
\Delta_{n}\left(\frac{z}{\sqrt{n}}\right)=1+\frac{\Delta_{n}^{\prime \prime}(0)}{2} \cdot \frac{z^{2}}{n}+O\left(n^{-2}\right)=1+\frac{g^{\prime}(\alpha) z^{2}}{8 n}+O\left(n^{-2}\right) .
$$

Thus, for any $c>1$ and all sufficiently large $n$,

$$
\operatorname{Esup}_{\beta \in B} Z_{n}^{1 / 2}(\beta) \leq\left(1-\frac{\left(\Psi^{\prime}(\alpha)-1 / \alpha\right) z^{2}}{8 n}+o\left(n^{-1}\right)\right)^{n} \leq c e^{-\left(\Psi^{\prime}(\alpha)-1 / \alpha\right) z^{2} / 8} .
$$

The proof is complete.

Lemma 3. For $u>0, g^{\prime}(u) / u+2 g^{\prime \prime}(u)>0$.

Proof. We use the method utilized e.g. in the proof of Lemma 1 in [3]. Consider the function

$$
q(u)=\frac{g^{\prime}(u)}{u}+2 g^{\prime \prime}(u)=-\frac{1}{u^{2}}-\frac{\Psi^{\prime}(u)}{u}-2 \Psi^{\prime \prime}(u), \quad u>0 .
$$

From the integral representations [1, formula (6.4.1)]

$$
\Psi^{\prime}(u)=\int_{0}^{\infty} \frac{t e^{-u t}}{1-e^{-t}} d t, \quad \Psi^{\prime \prime}(u)=-\int_{0}^{\infty} \frac{t^{2} e^{-u t}}{1-e^{-t}} d t
$$


and the evident relations

$$
\frac{1}{u}=\int_{0}^{\infty} e^{-u t} d t, \quad \frac{1}{u^{2}}=\int_{0}^{\infty} t e^{-u t} d t
$$

by the convolution theorem for Laplace transforms we get

$$
q(u)=\int_{0}^{\infty} e^{-u t} d(t) d t, \quad u>0
$$

where

$$
d(t)=-t+\frac{2 t^{2}}{1-e^{-t}}-\int_{0}^{t} \frac{v}{1-e^{-v}} d v, \quad t>0
$$

Differentiating yields

$$
d^{\prime}(t)=\frac{h(t)}{\left(e^{t}-1\right)^{2}}, \quad t>0
$$

where

$$
h(t)=3 t\left(e^{2 t}-e^{t}\right)-2 t^{2} e^{t}-\left(e^{t}-1\right)^{2}=(3 t-1) e^{2 t}-\left(2 t^{2}+3 t-2\right) e^{t}-1 .
$$

Making use of the series representation for $e^{t}$, we obtain

$$
h(t)=\sum_{k=3}^{\infty} \frac{c_{k} t^{k}}{k !}
$$

where

$$
c_{k}=2^{k-1}(3 k-2)+2-k-2 k^{2} .
$$

Since $c_{k}>0$ for any $k \geq 3$, we conclude that $d^{\prime}(t)>0$, and therefore, for any $t>0$,

$$
d(t)>\lim _{s \rightarrow 0} d(s)=0 .
$$

Thus, $q(u)>0$ for any $u>0$. The proof is complete.

\section{References}

[1] M. Abramovitz and I. A. Stegun (eds.), Handbook of Mathematical Functions with Formulas, Graphs and Mathematical Tables, Dover, New York, 1965.

[2] H. Alzer, On some inequalities for the gamma and psi functions, Math. Comp. 66 (1997), 373-389.

[3] - Inequalities for the gamma function, Proc. Amer. Math. Soc. 128 (1999), 141147.

[4] O. Barndorff-Nielsen, Information and Exponential Families in Statistical Theory, Wiley, New York, 1978.

[5] A. A. Borovkov, Mathematical Statistics, Nauka, Moscow, 1984 (in Russian).

[6] K. O. Bowman and L. R. Shenton, Properties of Estimators for the Gamma Distribution, Dekker, New York, 1988. 
[7] B. R. Crain, Exponential models, maximum likelihood estimation, and the Haar condition, J. Amer. Statist. Assoc. 71 (1976), 737-740.

[8] H. Dang and G. Weerakkody, Bounds for the maximum likelihood estimates in twoparameter gamma distribution, J. Math. Anal. Appl. 245 (2000), 1-6.

[9] J. Hájek, Z. Šidák and P. K. Sen, Theory of Rank Tests, 2nd ed., Academic Press, San Diego, 1999.

[10] I. A. Ibragimov and R. Z. Has'minskii, Statistical Estimation Asymptotic Theory, Springer, New York, 1981.

[11] E. L. Lehmann, Theory of Point Estimation, Wiley, New York, 1987.

[12] A. Nagaev, Limit theorems under testing hypotheses, UMK, Torun, 1996.

[13] A. Zaigraev and A. Podraza-Karakulska, On estimation of the shape parameter of the gamma distribution, Statist. Probab. Letters 78 (2008), 286-295.

Faculty of Mathematics and Computer Science

Nicolaus Copernicus University of Toruń

Chopina 12/18

87-100 Torun, Poland

E-mail: alzaig@mat.uni.torun.pl

agap@mat.uni.torun.pl

Received on 10.9.2007;

revised version on 22.2.2008 
